Full-length article

\title{
Dual functions of a monoclonal antibody against cell surface F1F0 ATP synthase on both HUVEC and tumor cells ${ }^{1}$
}

\author{
Xia ZHANG ${ }^{2,3}$, Feng GAO ${ }^{4}$, Li-li YU ${ }^{2,3}$, Yan PENG ${ }^{3,5}$, Hong-hai LIU $^{3}$, Jin-ying LIU $^{3}$, Ming YIN ${ }^{2,6}$, Jian NI ${ }^{3,6}$ \\ ${ }^{2}$ School of Pharmacy, Shanghai Jiao Tong University, Shanghai 200240, China; ${ }^{3}$ Human Antibodomics (Suzhou Industrial Park), Suzhou, \\ China; ${ }^{4}$ Shanghai Sixth People's Hospital of Shanghai, Shanghai 200233, China; ${ }^{5}$ Department of Biochemistry and Molecular Biology, \\ Second Military Medical University, Shanghai 200433, China
}

\section{Key words}

F1F0 ATP synthase; monoclonal antibody; doxorubicin; human umbilical vein endothelial cells; tube formation; cell migration

${ }^{1}$ This work was supported by the National High Technology Research and Development Program of China (863 Program, No 2007AA02Z180).

${ }^{6}$ Correspondence to Prof Jian NI and Prof Ming YIN.

Phn 86-21-3420-4737.

Fax 86-21-3420-4457.

E-mail jiannihome@yahoo.com (Jian NI).

E-mail myin@sjtu.edu.cn (Ming YIN).

Received 2008-02-28

Accepted 2008-05-19

doi: $10.1111 / \mathrm{j} .1745-7254.2008 .00830 . \mathrm{x}$

\begin{abstract}
Aim: To generate a monoclonal antibody (McAb) against cell surface F1F0 ATP synthase (ATPase) and observe its antitumoral activity on both human umbilical vein endothelial cells (HUVEC) and tumor cells. Methods: Hybridoma cells secreting McAb against ATPase were produced by polyethylene glycol-mediated fusions and screened by ELISA. The specificity of McAb was demonstrated by immunofluorescence and confocal imaging, as well as flow cytometry analysis. After the blockade of surface ATPase with McAb on HUVEC and human breast adenocarcinoma MDA-MB-231 cells, an ATP determination kit and CellTiter ${ }^{96}$ AQueous Assay (MTS) assay were used to detect the effect of the antibody on extracellular ATP modification and cell proliferation. A cellular cytotoxicity assay in combination with doxorubicin, and a cell migration assay on MDA-MB-231 cells were used to determine the antitumoral activity. Finally, a HUVEC tube formation assay was used to detect the antiangiogenic effect of McAb178-5G10. Results: A monoclonal antibody (McAb178-5G10) against the $\beta$-subunit of ATPase was generated, and its reactivity toward HUVEC and tumor cells was studied. We demonstrate that McAb178-5G10 binds to ATPase at the cell surface, where it is able to inhibit ATP synthesis. This antibody also prevents the proliferation of HUVEC and MDA-MB-231 cells. Furthermore, McAb178-5G10 enhances the tumoricidal effects of doxorubicin $(P<0.05)$, inhibits the migration of MDA-MB231 in transwell assays $(P<0.01)$, and disrupts HUVEC tube formation on Matrigel $(P<0.01)$. Conclusion: McAb178-5G10 binds preferentially to cell surface ATPase, blocks ATP synthesis, and exhibits both antiangiogenic and antitumorigenic effects.
\end{abstract}

\section{Introduction}

F1F0 ATP synthase (ATPase) is an enzymatic complex responsible for ATP synthesis. In mammals, this complex is composed of 17 subunits, 5 of which make up the easily detached F1 complex with the stoichiometry $\alpha_{3} \beta_{3} \gamma \delta \varepsilon$. The remaining subunits are components of 2 stalk domains and the proton pumping machinery, $\mathrm{F}^{[1]}$. Until recently, this complex was believed to be localized exclusively to mitochondria $^{[1]}$. However, current studies have demonstrated that components of ATPase exist on the outer surface of the plasma membrane, where their functions may include the regulation of intracellular $\mathrm{pH}$, cellular response to antiangiogenic agents, and cholesterol homeostasis ${ }^{[2]}$.

Identification of ATPase as the major angiostatin-binding site on the endothelial cell surface suggests that ATP metabolism may participate in the angiostatin response ${ }^{[3,4]}$. The inhibition of the endothelial cell surface ATPase using anti-ATPase antibodies mimicked the antiproliferative effects of angiostatin on endothelial cells $\mathrm{s}^{[3,5]}$. This result suggests that ATP synthesis and coupled proton transport may contribute to the maintenance of endothelial cell homeostasis ${ }^{[5,6]}$. ATPase is also present on the outer surface of many cancer cells, including lung, liver, and breast cancer cells ${ }^{[-8]}$. We have previously studied high metastatic hepatocellular car- 
cinoma cell (MHCC97-H) and low metastatic hepatocellular carcinoma cell (MHCC97-L) using 2-D protein fractionation ${ }^{[8,9]}$. A series of proteins related to tumor metastasis, including ATPase, was identified by matrix-assisted laser desorption/ionization time-of-flight mass spectrometry. These results indicate that ATPase may be useful as a novel cancer therapeutic target.

In the present study, we obtained a monoclonal antibody (McAb, McAb178-5G10) against cell surface ATPase and identified its reactivity toward human umbilical vein endothelial cells (HUVEC) and tumor cells. McAb178-5G10 binds preferentially to cell surface ATPase in both tumor and endothelial cells. We report a novel antibody that has both antitumoral and antiangiogenic capabilities, and may be further developed as a novel therapeutic treatment for tumors that overexpress ATPase.

\section{Materials and methods}

Cell culture Mammary epithelial cells MCF-10F (ATCC, CRL-10318) and breast cancer cells MDA-MB231(ATCC, HTB-26) were purchased from ATCC (Manassas, VA, USA). The MDA-MB-231 cells were cultured in Dulbecco's modified Eagle's medium (DMEM, Gibco, Grand Island, NY, USA) supplemented with $10 \%$ fetal bovine serum (Gibco, USA). MCF-10F cells were grown in a 1:1 ( $v / v)$ DMEM and Ham's F12 mixture (Gibco, USA), supplemented with $5 \%$ heat-inactivated horse serum (Gibco, USA). The HUVEC were obtained from human umbilical cord veins from normal pregnancies in non-smoking women immediately after delivery and cultured in endothelial cell medium (ECM, Sciencell, San Diego, CA, USA) up to the fifth passage before use. Sp2/0 mouse myeloma cells were cultured in RPMI-1640 medium (Gibco, USA) with $10 \%$ fetal bovine serum (FBS). All the cells were cultured with $100 \mathrm{U} / \mathrm{mL}$ penicillin (Gibco, USA) and 100 $\mathrm{mg} / \mathrm{mL}$ streptomycin (Gibco, USA) at $37{ }^{\circ} \mathrm{C}$ with $5 \% \mathrm{CO}_{2}$.

Antigen production and monoclonal antibody generation All animal studies were conducted in accordance with the guidelines of Shanghai Jiao Tong University Animal Care and Use Committee. Bovine heart F1 subunit of ATPase was obtained and purified from fresh bovine hearts ${ }^{[10]}$. The sequence encoding the ATPase $\beta$-subunit mature peptide was cloned from HUVEC and inserted into a prokaryotic expression vector pET28a (+) with a $6 \mathrm{X}$ His-tag at its N-terminus (Novagen, Madison, WI, USA). This construct was transformed into Escherichia coli BL21 (DE3) to induce its expression. The target protein was purified by $\mathrm{Ni}$-Sepharose FF metal-chelating chromatography (GE Healthcare, Waukesha, WI, USA) and refolded by rapid dilution. BALB/ c mice were obtained from the Shanghai Laboratory Animal Center of Chinese Academy of Sciences (Shanghai, China), and 8-12-week-old female mice were injected intradermally with the purified bovine heart F1 subunit of ATPase ${ }^{[11]}$. Splenocytes were used in polyethylene glycol-mediated fusions with Sp2/0 mouse myeloma cells. Hybridomas were obtained in 96-well tissue culture plates in RPMI-1640 supplemented with $20 \%$ FBS and HAT (hypoxanthine, aminopterin, thymidine). Cell supernatants were screened for antibodies binding to the bovine heart F1 subunit of ATPase. The positive clones were screened with the recombinant ATPase $\beta$ subunit by ELISA ${ }^{[12]}$. Subcloned cells in 96-well plates were recovered and injected into BALB/c mice $\left(1 \times 10^{6}\right.$ per mouse $)$ intraperitoneally. Antibodies in the ascites were purified by affinity chromatography with protein A-Sepharose CL-4B (GE Healthcare, USA). The immunoglobulin subtype of the McAb was determined using a mouse monoclonal antibody isotyping kit (Sigma, St. Louis, MO, USA) and its specificity was tested by Western blotting.

Affinity measurements The binding and dissociation kinetics of McAb178 with the recombinant ATPase $\beta$-subunit were determined using a BIAcore surface plasmon resonance instrument (Pharmacia, Uppsala, Sweden) as described $^{[22]}$. Briefly, $1400 \mathrm{RU}$ of the recombinant ATPase $\beta$ subunit $(25 \mu \mathrm{g} / \mathrm{mL}$ in $10 \mathrm{mmol} / \mathrm{L}$ sodium acetate, $\mathrm{pH} 4.5)$ were covalently bound through amino groups to a CM5 sensor chip. Association and dissociation of the McAb178$5 \mathrm{G} 10$ and $\mathrm{A} 21351$ (10 and $80 \mathrm{nmol} / \mathrm{L})$ were measured under continuous flow $(5 \mu \mathrm{L} / \mathrm{min})$. The determination of $k_{\text {on }}$ was done by plotting $(\ln [\mathrm{d} R / \mathrm{d} t]) / t$ versus concentration, whereas $k_{\text {off }}$ was determined from the dissociation part of the sensogram at the highest antibody concentration analyzed. Based on these kinetic measurements, the dissociation constant, $K_{d}$, and the association constant, $K_{a}$, were calculated.

Flow cytometry and confocal imaging of immunofluorescence For the flow cytometry assay ${ }^{[13]}$, the cells were detached from culture plates with $5 \mathrm{mmol} / \mathrm{L}$ ethylenediaminetetraacetic acid (EDTA, Gibco, USA) and washed twice with ice-cold phosphate buffered saline (PBS, pH 7. 4). The cells were incubated for $1 \mathrm{~h}$ with McAb178-5G10, A21351 (a monoclonal antibody against the ATPase $\beta$ subunit, Invitrogen, Eugene, OR, USA), or mouse IgG1 (an isotype control of McAb178-5G10, Invitrogen, USA) diluted in blocking solution [PBS with $5 \%$ bovine serum albumin (BSA), Calbiochem, San Diego, CA, USA]. The cells were washed again and incubated for $30 \mathrm{~min}$ with Alexa488 conjugated goat anti-mouse IgG (Invitrogen, USA) and washed 3 times. Propidium iodide (Becton Dickinson, 
Mountain View, CA, USA) was added before fluorescence was analyzed in a FACScalibur cytometer using CellQuest software (Becton Dickinson, USA). For the confocal imaging assay ${ }^{[14]}$, the cells were cultured on glass-based dishes (Asahi Glass, Yokohama, Jpn) for $24 \mathrm{~h}$, fixed with $4 \%$ paraformaldehyde/PBS for $5 \mathrm{~min}$, and then permeabilized with $0.1 \%$ Triton $\mathrm{X}-100 /$ Hepes/DMEM for $3 \mathrm{~min}$. Immunostaining was performed at $25{ }^{\circ} \mathrm{C}$ using the same antibodies and procedures as described for the flow cytometry assay. Nuclei were stained with 4'-6-diamidino2-phenylindole (DAPI, Sigma, USA), and sections were mounted with Prolong Gold antifade reagent (Invitrogen, USA) and observed by a confocal laser scanning microscopy system, FLUOVIEW FV1000 (Olympus, Tokyo, Jpn).

Cell surface ATP generation assay The cells (7000 per well) were equilibrated with serum-free medium at 37 ${ }^{\circ} \mathrm{C}$ with $5 \% \mathrm{CO}_{2}$ overnight, respectively, in 96-well plates. Then the cells were treated with different concentrations of McAb178-5G10, oligomycin (Sigma, USA), a known inhibitor of ATPase $\mathrm{F} 1$ or $\mathrm{mIgG} 1$ for $30 \mathrm{~min}$ at $37^{\circ} \mathrm{C}$ with $5 \% \mathrm{CO}_{2}$ to achieve $\mathrm{pH} 7.2$, or $17 \% \mathrm{CO}_{2}$ to achieve $\mathrm{pH} 6.7^{[6]}$. The cells were then incubated with adenosine diphosphate (Sigma, USA) for $60 \mathrm{~s}$, and supernatants were removed and assayed for ATP production using a bioluminescence assay kit (Invitrogen, USA) ${ }^{[15]}$. Samples were injected with the ATP assay mixture (Promega, Madison, WI, USA) and incubated for $10 \mathrm{~min}$ to stabilize the luminescence signal. Recordings were made in an Analyst HT (Molecular Devices, Sunnyvale, CA, USA) over a 20 s period. Data are expressed as moles of ATP per well based on standards determined under the same conditions during each experiment.

Cell proliferation and cellular cytotoxicity assay For the cell proliferation assay, the cells (1000 per well) were equilibrated with serum-free medium for $12 \mathrm{~h}$ at $37^{\circ} \mathrm{C}$ with $5 \%$ $\mathrm{CO}_{2}$ (17\% for acidic assay), respectively, in 96-well plates ${ }^{[6]}$. Then the cells were treated with various concentrations of McAb178-5G10, mIgG1, oligomycin, or medium alone for $48 \mathrm{~h}$. For the cytotoxicity assay, cells (10 000 per well) were equilibrated as described in the proliferation assay. The cells were treated with doxorubicin alone, McAb178-5G10, doxorubicin combined with McAb178-5G10 (40 $\mu \mathrm{g} / \mathrm{mL})$, doxorubicin combined with mIgG1 $(40 \mu \mathrm{g} / \mathrm{mL})$, doxorubicin combined with oligomycin $(10 \mu \mathrm{g} / \mathrm{mL})$, or medium alone for $24 \mathrm{~h}$. The number of living cells was measured using an MTS assay (Celltiter 96 Aqueous One solution cell proliferation assay, Promega, USA) ${ }^{[15]}$. Briefly, the MTS solution was added to each well and the cells were incubated for a final $3 \mathrm{~h}$. The absorbance at $490 \mathrm{~nm}$ was measured using a multiwell plate reader (Model 680, Harvard Apparatus, South Natick,
MA, USA), with wells containing medium, but with no cells serving as background.

Cell migration assay Migration assays ${ }^{[16]}$ were performed in transwell membrane filter inserts in 24-well tissue culture plates (Becton Dickinson, USA). The lower surface of the porous membrane was coated with human plasma fibronectin $(10 \mu \mathrm{g} / \mathrm{mL})$ at $4{ }^{\circ} \mathrm{C}$ overnight, and then blocked with $0.1 \%$ heat-inactivated BSA at $37{ }^{\circ} \mathrm{C}$ for $45 \mathrm{~min}$. The cells were added to inserts $\left(3 \times 10^{4}\right.$ cells/insert $)$ after preincubation with McAb178-5G10 (40 $\mu \mathrm{g} / \mathrm{mL}), \mathrm{mIgG1}(40$ $\mu \mathrm{g} / \mathrm{mL})$, oligomycin $(10 \mu \mathrm{g} / \mathrm{mL})$, or medium alone at $37^{\circ} \mathrm{C}$ for $30 \mathrm{~min}$. Migration was allowed to proceed at $37{ }^{\circ} \mathrm{C}$ for $8 \mathrm{~h}$. The cells were fixed, and cotton swabs were used to remove the cells that did not migrate through the filters. After fixation, the cells that had migrated to the lower surface of the porous membrane were stained with crystal violet and counted.

HUVEC tube formation assay Matrigel (Becton Dickinson, USA) was added to a 96 -well plate at $4{ }^{\circ} \mathrm{C}$ and allowed to gel for $2 \mathrm{~h}$ at $37^{\circ} \mathrm{C}(100 \mu \mathrm{L} / \text { well at } 15 \mathrm{mg} / \mathrm{mL})^{[17]}$. The cells $\left(5 \times 10^{4}\right)$ were incubated overnight in serum-free medium before detaching, and were then incubated with McAb178-5G10 (40 $\mu \mathrm{g} / \mathrm{mL}), \mathrm{mIgG1}(40 \mu \mathrm{g} / \mathrm{mL})$, oligomycin $(10 \mu \mathrm{g} / \mathrm{mL})$,or medium alone for $20 \mathrm{~min}$ at $37^{\circ} \mathrm{C}$. Endothelial cell migration and rearrangement were visualized after 4-6 h. Only points generating at least 3 tubules were counted.

Statistical analysis Data from several independent experiments were pooled and analyzed using a two-tailed Student's $t$-test. Statistical significance was assumed if $P<$ 0.05 . Representative fields of the migration assay and tube formation assay were photographed using a Olympus microscope $(\times 200)$.

\section{Results}

Antigen production and McAb178-5G10 generation We sought to generate $\mathrm{McAb}$ against the catalytic $\beta$-subunit of human F1F0 ATPase (ATP5B). The human ATP5B protein (accession No NP_001677) and bovine ATP5B protein (accession No NP_786990) have sequence homology ( 97\% positivity), generated by the NCBI Blastp ${ }^{[18]}$. In order to generate $\mathrm{McAb}$ against natural epitopes of the ATPase catalytic subunit, we first purified the F1 subunit of ATPase from bovine heart mitochondria. We used both this natural immunogen and the prokaryotic-expressed human ATPase $\beta$-subunit for screening. The F1 subunit (Figure 1A) of ATPase was purified and identified by SDS-PAGE. Coomassie blue staining showed 2 main bands, 1 for the ATPase $\alpha$-subunit $(\sim 55 \mathrm{kDa})$ and another for the ATPase $\beta$-subunit ( $\sim 50 \mathrm{kDa})$. The recombinant human ATPase $\beta$-subunit (His-tag: $\sim 55 \mathrm{kDa}$ ) 

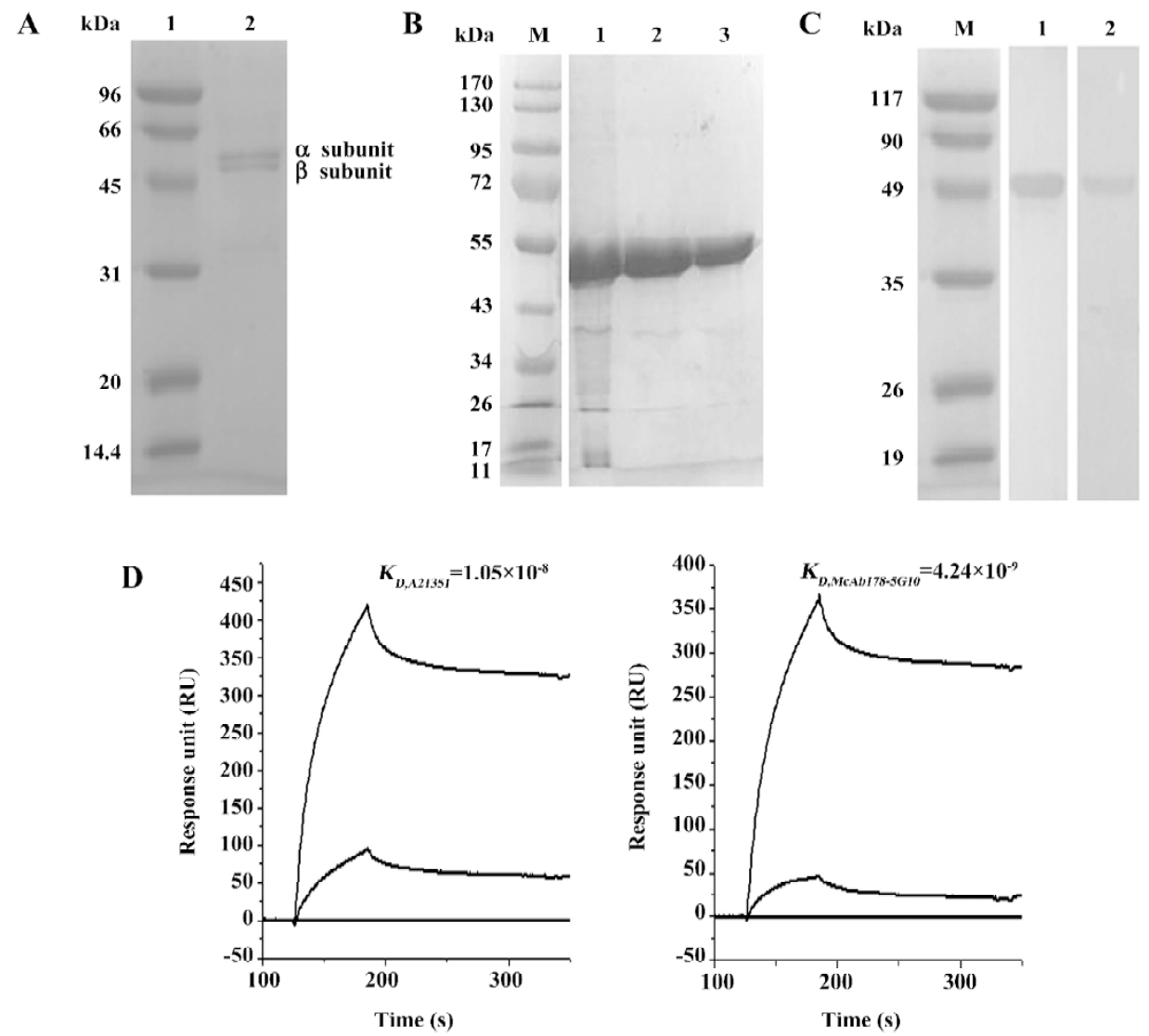

Figure 1. Antigen purification and antibody characterization. (A) Bovine heart F1 subunit was separated by SDS-PAGE. Lane 1 shows a protein marker (M0021, Huamei, China), and Lane 2 represents the F1 subunit, showing the $\alpha-$ subunit ( $55 \mathrm{kDa})$ and $\beta$-subunit ( 50 kDa). (B) Recombinant human ATP5B was separated by SDS-PAGE. Lane 1 represents induced BL21 (DE3) with pET28a (+)-ATP5B, lane 2 represents purified recombinant human ATP5B, lane 3 represents refolded recombinant human ATP5B. (C) Western blot analysis of HUVEC (lane 2) and MDA-MB-231(lane 3) cell lysates reacted with McAb178-5G10. Lane 1 shows prestained protein marker (49 kDa, P0066, Beyotime, China). (D) The affinity of McAb178-5G10 (a) and A21351 (b) to recombinant ATP synthase $\beta$ subunit were evaluated by BIAcore.

was expressed, purified, refolded, and tested by SDS-PAGE (Figure 1B). Twelve hybridomas were screened and only 1 was identified that recognized both the natural immunogen and the recombinant ATPase $\beta$-subunit (McAb178-5G10). The others may recognize the non-catalytic subunits of the ATPase F1, such as $\alpha, \delta$, or $\varepsilon$ subunits. The immunoglobulin subtype of McAb178-5G10 is immunoglobulin G 1 (IgG1). The Western blot analysis identified only a single band corresponding to the molecular mass of the ATPase $\beta$-subunit (Figure 1C). The affinity of McAb178-5G10 and A21351 to the recombinant ATPase $\beta$-subunit was evaluated by BIAcore. Dissociation constants were $K_{D, M c A b 178-}$ ${ }_{5 G 10}=4.24 \times 10^{-9}$ and $K_{D, A 2135 I}=1.05 \times 10^{-8}$ (Figure 1D).

McAb178-5G10 showed preferable binding to cell surface ATPase The subcellular localization of McAb178-5G10 and A21351 was examined by confocal immunofluorescence microscopy (Figure 2A). Indirect immunostaining of permeabilized cells with McAb178-5G10, A21351, or mIgG1 at the same concentration was used. Interestingly, McAb1785 G10 showed preferential binding to cell surface ATPase of HUVEC and MDA-MB-231 cells, while A21351 showed both intracellular and extracellular binding. To confirm the surface binding of the $\mathrm{McAb}$, indirect immunostaining of the intact cells was analyzed through flow cytometry ${ }^{[19]}$. In MDA-MB231 and HUVEC cells, the distribution of Alexa-488 fluorescence intensities obtained after incubation with McAb1785 G10 and A21351 showed an increase in comparison with mIgG1. MCF-10F showed almost no increase (Figure 2B). Thus the fluorescence increase observed in MDA-MB-231 cells and HUVEC is a result of the McAb specifically binding to the cell surface expressed as ATPase.

Inhibition of tumor cell surface ATP generation To examine the inhibitory effect of the antibody on ATP synthesis, a cell surface ATP generation assay was performed under 
A

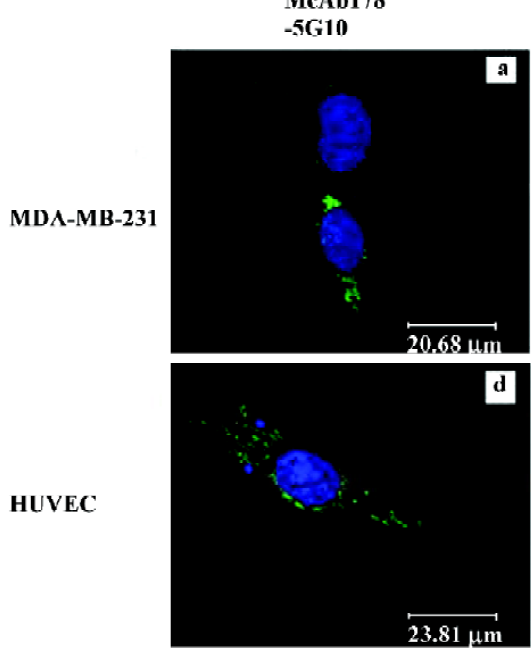

A21351
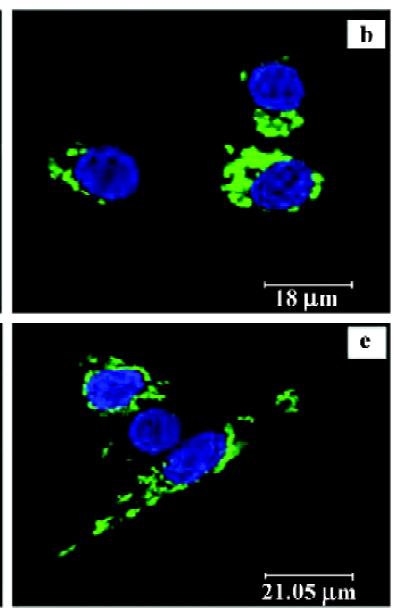

mIgG1

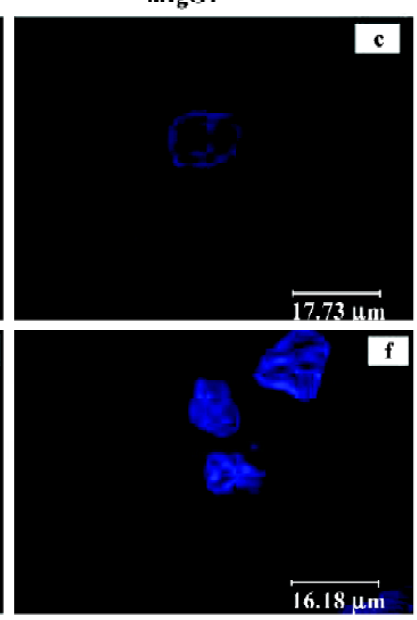

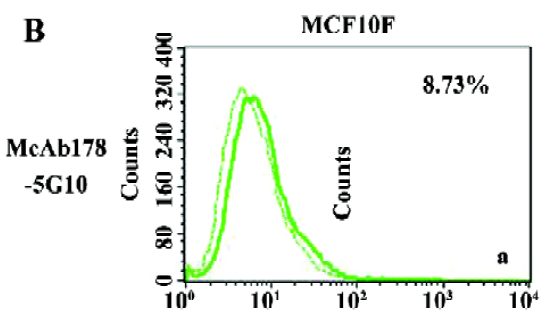
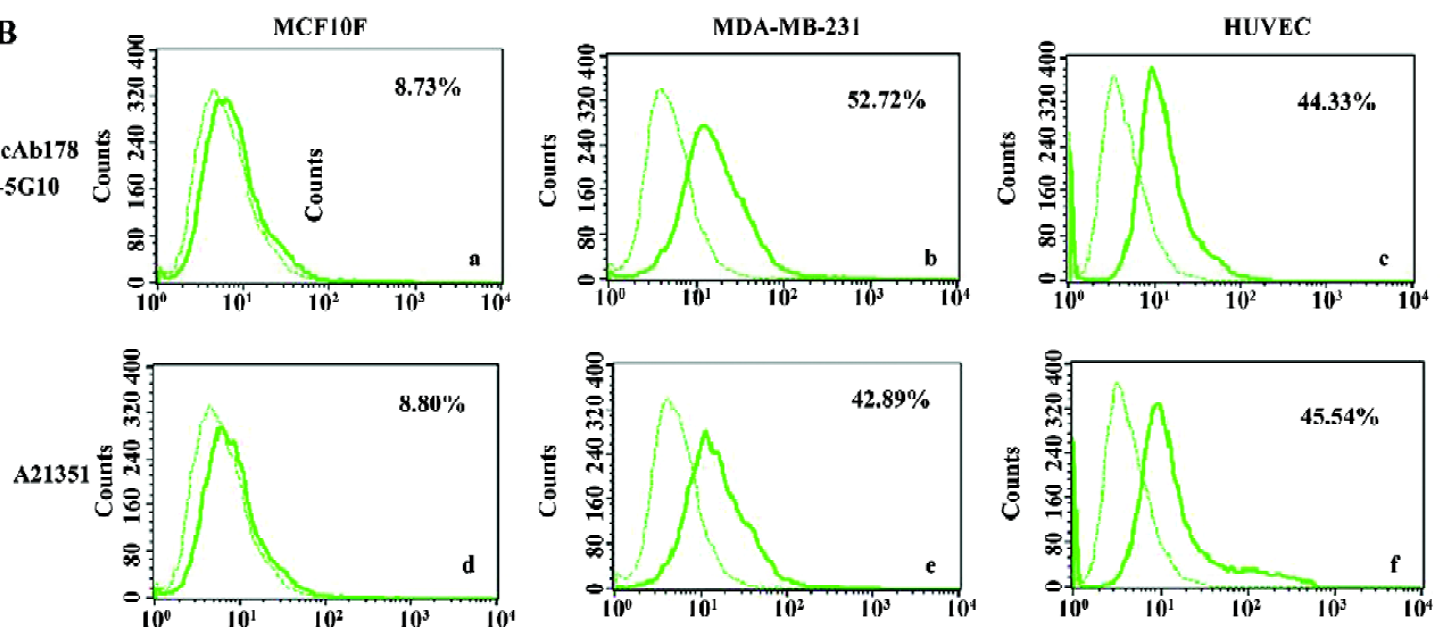

Figure 2. McAb178-5G10 bound preferentially to cell surface ATP synthase. (A) Confocal imaging was used for McAb178-5G10 (a, d), A21351 (b, e) binding on cells. mIgG1 (c, f) was an isotype control. (B) Histogram plots of flow cytometry are shown for the binding of McAb178-5G10 (a, b, c) and A21351 (d, e, f) on cells, where solid lines represent cells incubated with antibody, and dashed lines represent IgG1 negative control.

both neutral and acidic conditions. We found that McAb1785 G10 inhibited ATP synthesis at the surface of MDA-MB231 (Figure 3A) and HUVEC (Figure 3B) cells in a dose-dependent manner. mIgG1 showed no inhibition at all. Oligomycin, a known inhibitor of the F1 protein, also showed dose-dependent inhibition, confirming the reliability of this test. The inhibitory effect by McAb178-5G10 increased under acidic conditions (50\% inhibition concentration, $\mathrm{IC}_{50}=68.9$ $\mu \mathrm{g} / \mathrm{mL}$ for MDA-MB-231 and $61.5 \mu \mathrm{g} / \mathrm{mL}$ for HUVEC) compared with neutral conditions $\left(\mathrm{IC}_{50}=203.7 \mu \mathrm{g} / \mathrm{mL}\right.$ for MDAMB-231 and 107.0 $\mu \mathrm{g} / \mathrm{mL}$ for HUVEC), while oligomycin had an equal effect under both conditions $\left(\mathrm{IC}_{50}=23.8,28.3 \mu \mathrm{g} / \mathrm{mL}\right.$ under acidic conditions and 22.8 and $27.2 \mu \mathrm{g} / \mathrm{mL}$ under neu- tral conditions). The maximal inhibition of McAb178-5G10 to MDA-MB-231 and HUVEC was $~ 55 \%$ and $64 \%(150 \mu \mathrm{g} / \mathrm{mL}$ under acidic conditions), and the maximal inhibition of oligomycin to both the cells was $\sim 100 \%(150 \mu \mathrm{g} / \mathrm{mL}$ under both acidic and neutral conditions). However, differences of ATP generation at the surface of MCF-10F cells were undetectable between treated and untreated wells (data not shown).

Inhibition of cell proliferation and assessment of cellular cytotoxicity To observe the consequence of blockage of the cell surface ATPase using our antibody, a proliferation assay was performed under different external $\mathrm{pH}$ conditions. McAb178-5G10 showed a little inhibition of proliferation in MDA-MB-231 (Figure 3C) and HUVEC cells exposed to acidic 

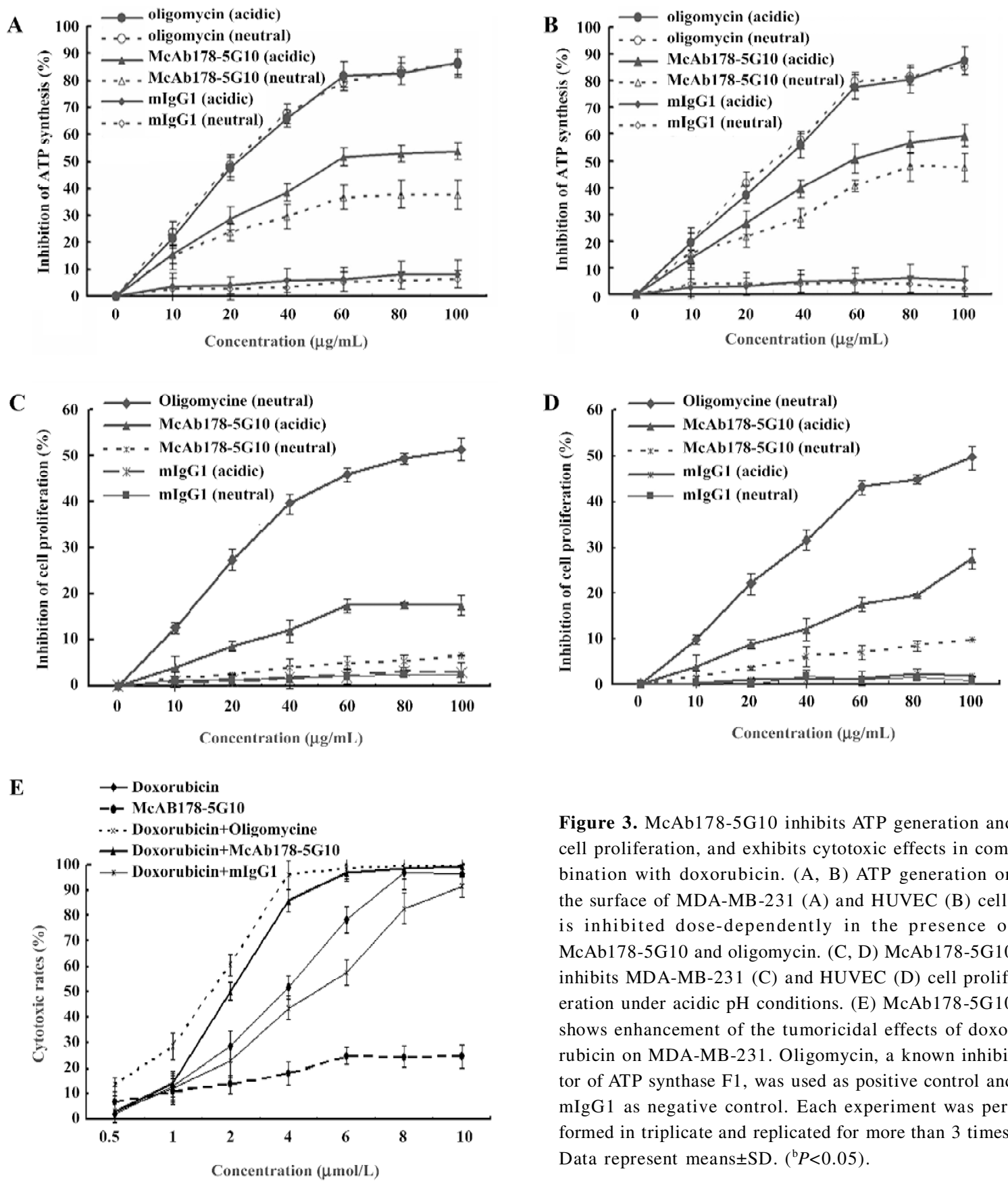

Figure 3. McAb178-5G10 inhibits ATP generation and cell proliferation, and exhibits cytotoxic effects in combination with doxorubicin. (A, B) ATP generation on the surface of MDA-MB-231 (A) and HUVEC (B) cells is inhibited dose-dependently in the presence of McAb178-5G10 and oligomycin. (C, D) McAb178-5G10 inhibits MDA-MB-231 (C) and HUVEC (D) cell proliferation under acidic $\mathrm{pH}$ conditions. (E) McAb178-5G10 shows enhancement of the tumoricidal effects of doxorubicin on MDA-MB-231. Oligomycin, a known inhibitor of ATP synthase F1, was used as positive control and $\mathrm{mIgG1}$ as negative control. Each experiment was performed in triplicate and replicated for more than 3 times. Data represent means \pm SD. $\left({ }^{b} P<0.05\right)$.

$\mathrm{pH}\left(\mathrm{IC}_{50}=850.9 \mu \mathrm{g} / \mathrm{mL}\right.$ for MDA-MB-231 and $341.5 \mu \mathrm{g} / \mathrm{mL}$ for HUVEC), but there was no inhibition at neutral $\mathrm{pH}$ (Figure 3D). This $\mathrm{pH}$-dependent inhibition was in agreement with a previous report that ATPase inhibitors prevent HUVEC cell surface ATP generation to a greater degree under conditions of acidosis ${ }^{[3,5,6]}$. The maximal inhibition of McAb178-5G10 to MDA-MB-231 and HUVEC was $22 \%$ and $37 \%(150 \mu \mathrm{g} / \mathrm{mL}$ under acidic conditions), and the maximal inhibition of oligomycin to both the cells was $\sim 76 \%(150 \mu \mathrm{g} / \mathrm{mL}$ under both acidic and neutral conditions). mIgG1 exhibited no effect on

the proliferation of MDA-MB-231 and HUVEC cells at either of the $\mathrm{pH}$ conditions tested. MCF-10F showed no differences between treated and untreated wells (data not shown). To further examine the function of McAb178-5G10 on breast cancer cells, a cytotoxicity assay was performed in the presence of the chemotherapeutic, doxorubicin. Interestingly, we found the $\mathrm{IC}_{50}$ of doxorubicin on MDA-MB-231 decreased $(P<0.05)$ when combined with $\mathrm{McAb178}-5 \mathrm{G} 10\left(\mathrm{IC}_{50}=2.7 \mu \mathrm{mol} / \mathrm{L}\right.$ alone and $\mathrm{IC}_{50}=1.8 \mu \mathrm{mol} / \mathrm{L}$ when combined), as well as the positive control oligomycin $\left(\mathrm{IC}_{50}=1.3 \mu \mathrm{mol} / \mathrm{L}\right.$ when 
combined), while $\mathrm{mIgG} 1\left(\mathrm{IC}_{50}=3.7 \mu \mathrm{mol} / \mathrm{L}\right.$ combined $)$ showed no effect on the sensitivity of MDA-MB-231 cells to doxorubicin (Figure 3E). A21351, which binds to the inner mitochondrial membrane, showed no inhibitory effect to both MDA-MB-231 and HUVEC cells (data not shown).

Inhibition of MDA-MB-231 cell migration To investigate whether cell surface ATPase activity is a requirement for cell migration, we tested MDA-MB-231 cell migration in the presence or absence of McAb178-5G10. The effect on cell migration was assessed by using a fibronectin-coated transwell assay. McAb178-5G10, as well as the positive control, oligomycin, quenched more than $75 \%$ of the migration of MDA-MB-231 cells compared with medium alone $(P<0.01)$, while $\mathrm{mIgG1}$ showed no influence at all (Figure 4$)$.

Inhibition of HUVEC tube formation To investigate the effect of McAb178-5G10 on the behavior of HUVEC during the process of angiogenesis in vitro, a matrigel tube formation assay was performed. HUVEC pretreated with McAb1785G10, as well as the positive control, oligomycin, showed clear disruption of tube formation compared with medium alone $(P<0.01)$, while $\mathrm{mIgG1}$ showed no effect at all (Figure 5).

\section{Discussion}

In this study, we provide evidence that McAb178-5G10 binds preferentially to the cell surface ATPase of tumor and endothelial cells, McAb178-5G10 inhibits ATP generation on the surface of tumor and endothelial cells under acidic $\mathrm{pH}$ conditions, McAb178-5G10 inhibits cell proliferation and migration, and decreases the $\mathrm{IC}_{50}$ of doxorubicin on MDAMB-231 $(P<0.01)$, and McAb178-5G10 inhibits HUVEC pro- liferation and disrupts tube formation. We therefore report the production of an antibody that exerts both anti-angiogenic and antitumoral activities, and may further be developed as a novel therapeutic agent for the treatment of tumors that overexpress ATPase.

Observations of cell surface ATPase are increasingly being reported in different types of cells. In addition to endothelial cells, ATPase or its subunits have now been localized to the tumor cell surface of HepG2 hepatocellular carcinoma, H1299 non-small cell lung cancer, K562 erythroleukemia, and A549 adenocarcinoma ${ }^{[16-18]}$. We have found that ATPase was present on the surface of MDA-MB231 cells, with comparable expression in HUVEC.

Cell surface ATPase activity is essential to tumor cell proliferation, migration, and HUVEC tube formation. The origin of cell surface ATPase has yet to be determined. In the present study, McAb178-5G10 selectively recognizes ATPase at the cell surface, rather than the inner mitochondrial membrane. Although $\alpha$ - and $\beta$-subunits have been observed on several tumor cell lines, studies have observed that these components were sometimes separately detected, raising the possibility of structurally different complexes, or alternatively, a differential masking of epitopes by uncharacterized structures ${ }^{[19]}$. The structure displayed on the cell surface may use some of its components to perform different functions ${ }^{[19]}$. It is now clear that post-translational modifications can alter the antigenic properties of proteins ${ }^{[20]}$. It is possible that either different post-translational modifications between the synthases or undetected isoforms of the human ATPase $\beta$-subunit exist.
A

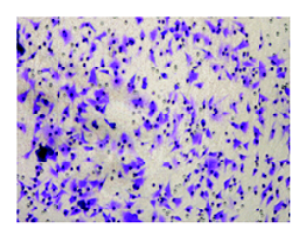

Medium control

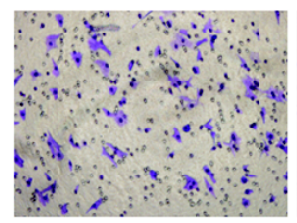

McAb178-5G10

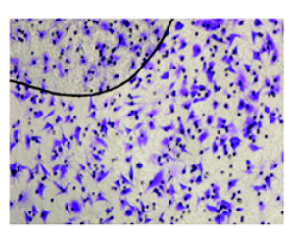

mIgG1 isotype control

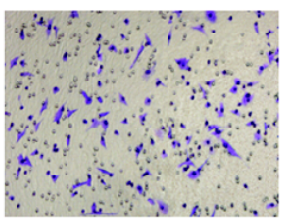

Oligomycin

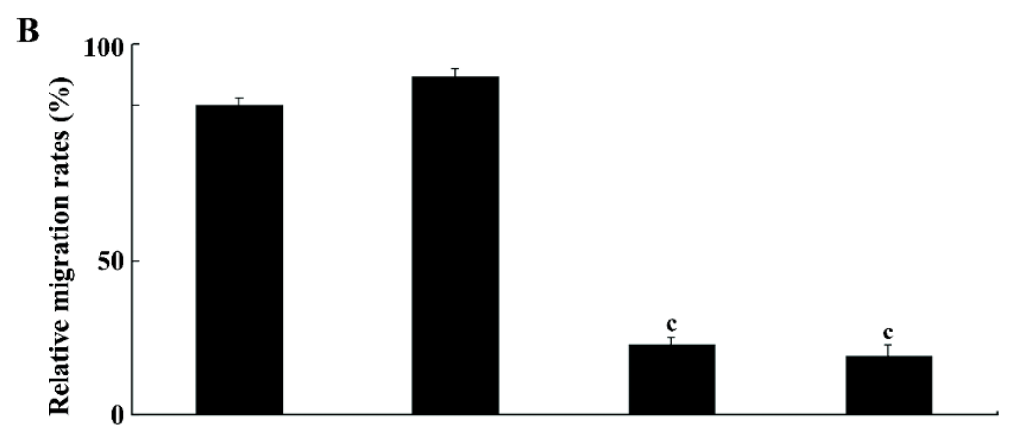

Figure 4. McAb178-5G10 inhibits cell migration. (A) Representative images of MDA-MB-231 cells migrating to the lower surface of the membrane in the presence of McAb178-5G10, mIgG1, or medium only. (B) Relative rates analysis of the experiments shown in (A) obtained by counting the number of cells migrating to the lower surface of the membrane. Oligomycin, a known inhibitor of ATP synthase F1, was used as positive control and $\mathrm{mIgG} 1$ as negative control. Each experiment was performed in triplicate and replicated for more than 3 times. Data represent means $\pm \mathrm{SD}$. $\left({ }^{\mathrm{c}} P<0.01\right)$. 
A

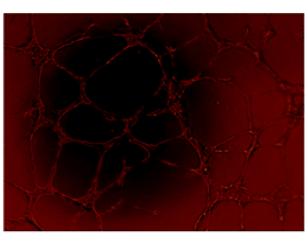

Medium control

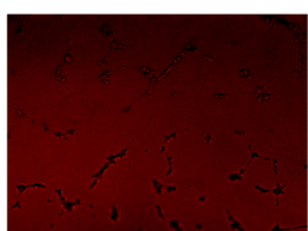

McAb178-5G10

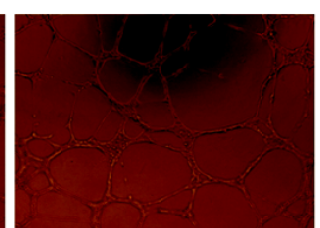

mIgG1 isotype control

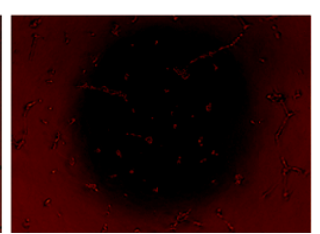

Oligomycin

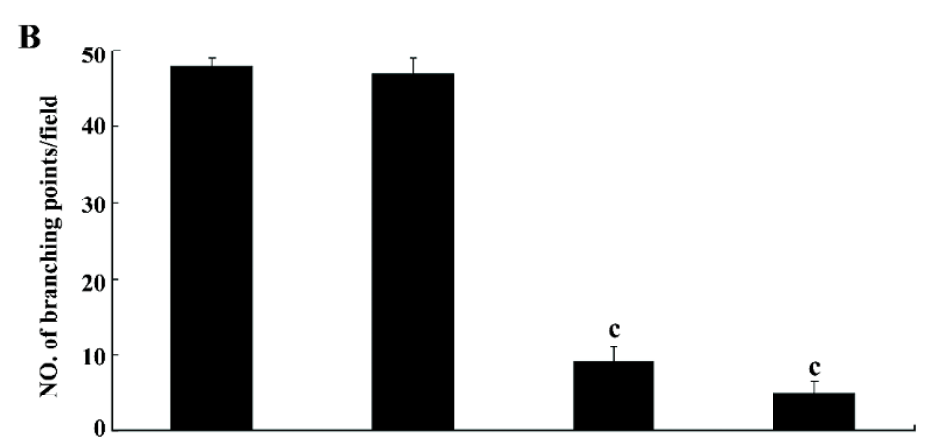

Figure 5. McAb178-5G10 inhibits tube formation (A) Representative images of HUVEC tube formation on Matrigel in the presence of McAb178-5G10, mIgG1, or medium only. (B) quantitative analysis of the experiments shown in (A) obtained by counting the number of branching points. Oligomycin, a known inhibitor of ATP synthase F1, was used as positive control and mIgG1 as negative control. Each experiment was performed in triplicate and replicated for more than 3 times. Data represent means \pm SD. $\left({ }^{c} P<0.01\right)$.

While the synthase could be generating ATP at the cell surface through well-established mechanisms, it is also possible that proton transport or endocytosis depend on this enzyme $^{[2]}$. The function of cell surface synthase has been identified in hepatocytes where it acts as the high-density lipoprotein (HDL) receptor through binding of apolipoprotein A-I and regulates lipoprotein internalization ${ }^{[21]}$ on endothelial cells. In these cells, surface ATPase binds angiostatin, regulates surface ATP levels, and modulates endothelial cell proliferation and differentiation ${ }^{[3,4]}$. On the surface of cancer cells, it is recognized by the antigen receptor of circulating cytotoxic lymphocytes of the $\gamma$ and $\delta$-subtype, and thus promotes innate tumor cell recognition and lysis ${ }^{[22]}$. The downstream effects of cell surface synthase remain to be determined. Here, we report that McAb178-5G10 modifies the extracellular ATP level, enhances the antitumoral activity of doxorubicin against MDA-MB-231 breast cancer cells, and inhibits cell migration. The mechanism of those functions, including modification of the extracellular ATP level, necessitates further investigation.

Angiostatin is a naturally occurring angiogenesis inhibitor produced in cancer patients ${ }^{[23]}$. Identification of ATPase as the major angiostatin-binding site on the endothelial cell surface suggests that ATPase plays an important role in the angiostatin response ${ }^{[4]}$. It has recently been reported that ectopically localized ATPase could help maintain neutral $\mathrm{pH}_{\mathrm{i}}$ under acidic condition ${ }^{[19]}$. Angiostatin could affect the regulation of $\mathrm{pH}_{\mathrm{i}}$ and have a profound effect on in vitro tube formation by endothelial cells ${ }^{[2]}$. Angiostatin is cytotoxic to tumor cells and inhibits endothelial cell proliferation ${ }^{[22]}$. Since angiostatin is relatively unstable with a short half-life in sera
(15 min), the identification of alternative inhibitors of ATPase is of great interest ${ }^{[24]}$. A recent publication by Chi has shown that a McAb against the ATPase $\beta$-subunit could bind to intracellular and extracellular ATPase in HUVEC and disrupts tube formation at an acidic $\mathrm{pH}^{[5]}$. Here, we have shown that McAb178-5G10 has an inhibitory effect on HUVEC, indicating angiostatin-like activity. These data as a whole suggest that ATPase present at the cell membrane in both tumor cells and HUVEC, and it might play important roles in both tumor metastasis and angiogenesis. Antibodies against it could potentially be used to prevent angiogenesis-mediated tumor growth and metastasis of cancers.

Although the function of cell surface ATPase requires further study, this study implies that it plays important roles in tumor cell migration and drug resistance, as well as endothelial cell migration and rearrangement. A McAb against cell surface F1F0 ATPase was obtained, and its antitumoral activity on both HUVEC and tumor cells was identified.

\section{Acknowledgements}

The authors acknowledge all the colleagues from the Neuropharmacology Laboratory of Shanghai Jiao Tong University (Shanghai, China) and Human Antibodomics (Suzhou Industrial Park, Suzhou, China) for their help with experimental techniques.

\section{Author contributions}

Xia ZHANG, Feng GAO, Yan PENG, Ming YIN, and Jian NI designed research; Xia ZHANG, Li-li YU performed research; Feng GAO, Jin-ying LIU, Hong-hai LIU contrib- 
uted new analytical reagents and tools; Xia ZHANG, Jian NI analyzed data; Xia ZHANG, Ming YIN wrote the paper.

\section{References}

1 Cross RL. Molecular motors: turning the ATP motor. Nature 2004; 427: 407-8.

2 Chi SL, Pizzo SV. Cell surface F0F1 ATP synthase: a new paradigm? Ann Med 2006; 38: 429-38.

3 Moser TL, Kenan DJ, Ashley TA, Roy JA, Goodman MD, Misra UK, et al. Endothelial cell surface F1-F0 ATP synthase is active in ATP synthesis and is inhibited by angiostatin. Proc Natl Acad Sci USA 2001; 98: 6656-61.

4 Moser TL, Stack MS, Asplin I, Enghild JJ, Højrup P, Everitt L, et al. Angiostatin binds ATP synthase on the surface of human endothelial cells. Proc Natl Acad Sci USA 1999; 96: 2811-6.

5 Chi SL, Wahl ML, Mowery YM, Shan S, Mukhopadhyay S, Hilderbrand SC, et al. Angiostatin-like activity of a monoclonal antibody to the catalytic subunit of F1F0 ATP synthase. Cancer Res 2007; 67: 4716-24.

6 Chi SL, Pizzo SV. Angiostatin is directly cytotoxic to tumor cells at low extracellular $\mathrm{pH}$ : a mechanism dependent on cell surfaceassociated ATP synthase. Cancer Res 2006; 66: 875-82.

7 Zhao Y, Zhang W, Kho Y, Zhao Y. Proteomic analysis of integral plasma membrane proteins. Anal Chem 2004; 76: 1817-23.

8 Li Y, Tang Y, Ye L, Liu B, Liu K, Chen J, Xue Q. Establishment of a hepatocellular carcinoma cell line with unique metastatic characteristics through in vivo selection and screening for metastasis-related genes through cDNA microarray. J Cancer Res Clin Oncol 2003; 129: 43-51.

9 Peng Y, Zhang X, Wang K, Jiang P, Jiao BH, Ni J. Proteome analysis of hepatocellular carcinoma cell lines with different metastasis potentials by two-dimensional liquid chromatography. World J Digestol 2007; 15:1482-7.

10 Aggeler R, Coons J, Taylor SW, Ghosh SS, Garcia JJ, Capaldi RA, et al. A functionally active human F1F0 ATPase can be purified by immunocapture from heart tissue and fibroblast cell lines, Subunit structure and activity studies. J Biol Chem 2002; 277: 33 906-12.

11 Sakaguchi N, Kimura T, Matsushita S, Fujimura S, Shibata J, Araki $\mathrm{M}$, et al. Generation of high-affinity antibody against $\mathrm{T}$ celldependent antigen in the Ganp gene-transgenic mouse. J. Immunol 2005; 174: 4485-94.

12 Köhler G, Milstein C. Continuous cultures of fused cells secreting antibody of predefined specificity. Nature $1975 ; 256: 495-7$.

13 Bueno C, Rodriguez-Caballero A, García-Montero A, Pandiella A, Almeida J , Orfao A. A new method for detecting TNF-alphasecreting cells using direct-immunofluorescence surface membrane stainings. J Immunol Methods 2002; 264: 77-87.

14 Zucker RM, Jeffay SC. Confocal laser scanning microscopy of whole mouse ovaries: excellent morphology, apoptosis detection, and spectroscopy. Cytometry A 2006; 69: 930-9.

15 Burwick NR, Wahl ML, Fang J, Zhong Z, Moser TL, Li B, et al. An inhibitor of the F1 subunit of ATP synthase (IF1) modulates the activity of angiostatin on the endothelial cell surface. J Biol Chem 2005; 280: 1740-5.

16 Lukong KE, Richard S. Breast tumor kinase BRK requires kinesin2 subunit KAP3A in modulation of cell migration. Cell Signal 2008; 20: 432-42.

17 Guo K, Li J, Wang H, Osato M, Tang JP, Quah SY, et al. PRL-3 initiates tumor angiogenesis by recruiting endothelial cells in vitro and in vivo. Cancer Res 2006; 66: 9625-35.

18 Kirley TL. Complementary DNA cloning and sequencing of the chicken muscle ecto-ATPase: Homology with the lymphoid cell activation antigen CD39. J Biol Chem 1997; 272: 1076-81.

19 Scotet E, Martinez LO, Grant E, Barbaras R, Jeno P, Guiraud M, et al. Tumor recognition following Vgamma9Vdelta2 $\mathrm{T}$ cell receptor interactions with a surface F1-ATPase-related structure and apolipoprotein A-I. Immunity 2005; 22: 71-80.

20 Cloos P A, Christgau S. Post-translational modifications of proteins: implications for aging, antigen recognition, and autoimmunity. 2004; 5: 139-58.

21 Martinez LO, Jacquet S, Esteve JP, Rolland C, Cabezón E, Champagne E, et al. Ectopic beta-chain of ATP synthase is an apolipoprotein A-I receptor in hepatic HDL endocytosis. Nature 2003; 421: 75-9.

22 Das B, Mondragon MO, Sadeghian M, Hatcher VB, Norin AJ. A novel ligand in lymphocyte-mediated cytotoxic: expression of the beta-subunit of $\mathrm{H}+$ transporting ATP synthase on the surface of tumor cell lines. J Exp Med 1994; 180: 273-81.

23 O'Reilly MS, Holmgren L, Shing Y, Chen C, Rosenthal RA, Moses $\mathrm{M}$, et al. Angiostatin: a novel angiogenesis inhibitor that mediates the suppression of metastases by a Lewis lung carcinoma. Cell 1994; 79: 315-28.

24 Kenan1 DJ, Wahl ML. Ectopic localization of mitochondrial ATP synthase: a target for anti-angiogenesis intervention? J Bioenerg Biomembr 2005; 37: 461-5. 